\title{
CDR1as/miR-7/CKAP4 axis contributes to the pathogenesis of abdominal aortic aneurysm by regulating the proliferation and apoptosis of primary vascular smooth muscle cells
}

\author{
FENG ZHAO*, TONGYUN CHEN ${ }^{*}$ and NAN JIANG \\ Department of Cardiac Surgery, Tianjin Chest Hospital, Tianjin 300222, P.R. China
}

Received June 16, 2019; Accepted February 13, 2020

DOI: $10.3892 /$ etm.2020.8622

\begin{abstract}
Abdominal aortic aneurysm (AAA) is characterized as dilation of the aortic wall. Dysregulation of vascular smooth muscle cells (VSMCs) can contribute to the development of this phenotype. Circular RNAs and microRNAs (miRNAs) can regulate the proliferation and apoptosis of VSMCs. This present study aimed to identify the mechanisms of action behind the regulation of cerebellar degeneration-related protein 1 antisense RNA (CDR1as)/miRNA (miR)-7 in VSMCs. The expression levels of miR-7 were upregulated, whereas the levels of CDR1as and cytoskeleton-associated protein 4 (CKAP4) were downregulated in aortic specimens obtained from 10 patients who underwent surgery for AAA compared with aortic specimens from 10 control patients who underwent coronary artery bypass surgery. The molecular mechanism of action of CDR1as/miR-7 was investigated in primary VSMCs. The results of Cell Counting kit-8 and cell growth curve assays revealed that overexpression of CDRlas and knockdown of miR-7, increased VSMC proliferation, whereas knockdown of CDRlas and overexpression of miR-7 suppressed VSMC proliferation. In addition, overexpression of CDR1as and knockdown of miR-7, suppressed apoptosis in VSMCs, indicated by the decreased levels of reactive oxygen species (ROS) and lactate dehydrogenase (LDH) activity, whereas knockdown of CDR1as and overexpression of miR-7 exhibited the opposite effects. The results of luciferase reporter and biotin pull-down assays confirmed that CDR1as directly bound to miR-7 and suppressed its expression. Additionally, the CDR1as-induced proliferation and suppressed apoptosis was reversed by the overexpression of miR-7. Furthermore, luciferase reporter, reverse transcription-quantitative PCR and western blot assays revealed that miR-7 directly targeted CKAP4 and
\end{abstract}

Correspondence to: Dr Nan Jiang, Department of Cardiac Surgery, Tianjin Chest Hospital, 261 South Tai-Erzhuang Road, Jinnan, Tianjin 300222, P.R. China

E-mail: nanjiang419@hotmail.com

${ }^{*}$ Contributed equally

Key words: vascular smooth muscle cells, apoptosis, microRNA-7, cytoskeleton associated protein $4, \mathrm{CDR} 1$ antisense RNA suppressed its expression. Additionally, the miR-7-suppressed proliferation and increased ROS and LDH activity were reversed by the overexpression of CKAP4. CDR1as also decreased caspase 3/7 activity, which was reversed by miR-7 mimics. miR-7 increased the activity of caspase $3 / 7$, which was again reversed by the overexpression of CKAP4. Therefore, CDRlas, miR-7 and CKAP4 may act in the same pathway to regulate VSMC proliferation and apoptosis.

\section{Introduction}

Abdominal aortic aneurysm (AAA) is a disease characterized by the dilation of the aorta that mostly affects adult patients, especially those $>65$ years (1). AAA can be life-threatening when an acute rupture occurs (2). Histological studies have revealed that AAA is associated with the impairment of apoptotic and inflammatory functions of vascular smooth muscle cells (VSMCs). VSMCs are the key source of elastin, which provides the elasticity of the aortic wall (3). VSMC apoptosis can reduce the levels of elastin, leading to the expansion of the aortic wall and ultimately resulting in alterations to the elasticity and remodeling of the abdominal aortic wall (4).

Non-coding RNAs (ncRNAs) are a class of non-protein coding RNAs that participate in numerous cellular processes and pathological conditions. This class includes microRNAs (miRNAs/miRs) and circular RNAs (circRNAs), among others (5). miRNAs have been demonstrated to be major post-transcriptional regulators of gene expression (6) and serve critical functions in vascular biology (7).

circRNAs interact with other molecules or miRNAs to regulate gene expression at the post-transcriptional or transcriptional levels (8), thus modulating various biological processes such as cell proliferation, apoptosis, invasion and migration (9). Cerebellar degeneration-related protein 1 antisense RNA (CDRlas) contains $>70$ binding sites to sponge miR-7 and modulate its activity on target genes (10). The CDR1as/miR-7 axis has been investigated in several types of cancer, such as esophageal squamous cell carcinoma and colorectal carcinoma (11). However, the functions of the CDR1as/miR-7 axis in AAA have not been investigated in detail.

Cytoskeleton-associated protein 4 (CKAP4), also termed p63 or cytoskeleton-linking membrane protein of $63 \mathrm{kDa}$, is a plasma membrane protein presented by VSMCs (12). Previous studies have suggested that CKAP4 may promote 
tumor progression through the PI3K/AKT (12) and cyclin B signaling pathways (13). CKAP4 and PI3K can form a complex at the proline-rich domain of CKAP4 and the Src homology 3 domain, which accelerates the production of phosphatidylinositol-3,4,5-triphosphate, leading to the activation of AKT and the proliferation of normal and cancer cells (13). Furthermore, the upregulation of CKAP4 increases the levels of cyclin B1 and cyclin $B 2$, which in turn decreases cell population at the $G_{2} / M$ phase and increases cell population at the $S$ phase, leading to the progression of clear cell renal cell carcinoma (14).

Previous studies have demonstrated that CDR1as can sponge miR-7 in osteosarcoma and hepatocellular carcinoma $(11,15)$; therefore, the present study used bioinformatics analysis to predict that CKAP4 may be targeted by miR-7. The aim of the present study was to determine the relationship among CDR1as, miR-7 and CKAP4 in the pathogenesis of AAA, which may ultimately serve as a potential treatment target for AAA.

\section{Materials and methods}

Patient tissues. Aortic specimens were collected from 10 patients who underwent ascending aortic aneurysm surgery (mean age, 63.25 \pm 6.24 ; men, 7; women, 3) and from 10 control patients who underwent coronary artery bypass graft

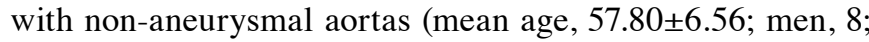
women, 2) between January 2017 and December 2018 at Tianjin Chest Hospital. Patients with bicuspid aortic valves or hereditary connective tissue disorders were excluded from the control group. The obtained aortic specimens were split into two parts, which were snap-frozen in liquid nitrogen for RNA isolation or primary VSMCs isolation. All patients signed written informed consent, and ethical approval was obtained from the Institutional Review Board of Tianjin Chest Hospital.

Cell culture and transfection. Primary VSMCs were isolated from the aforementioned 10 clinical aortic specimens who underwent ascending aortic aneurysm surgery as previous described with modifications (16). Following removal of fat and connective tissue, the tissues were cut into $3-\mathrm{mm}$ long sections and rubbed to remove the endothelium, mixed the 10 samples together, followed by incubation with $0.2 \%$ collagenase at $37^{\circ} \mathrm{C}$ and centrifugation at $120 \mathrm{xg}$ for $15 \mathrm{~min}$ at $4^{\circ} \mathrm{C}$ to obtain VSMCs. Following washing with PBS, the obtained pelleted VSMCs were seeded into six-well plates containing medium 231 (cat. no. M231500; Life Technologies; Thermo Fisher Scientific, Inc.) containing 5\% Smooth Muscle Growth Supplement (cat. no. S00725; Life Technologies; Thermo Fisher Scientific, Inc.), $100 \mathrm{U} / \mathrm{ml}$ penicillin and streptomycin (cat. no. 15140122; Life Technologies; Thermo Fisher Scientific, Inc.), and incubated in under $5 \% \mathrm{CO}_{2}$ at $37^{\circ} \mathrm{C}$ for $24 \mathrm{~h}$ before transfection.

All the mimics and vectors were purchased from GeneCopoeia, Inc. For transfections, the miR-7 mimics (5'-UGG AAGACUAGUGAUUUUGUUGU-3'), miR-negative control (mimics NC, 5'-CAGUACUUUUGUGUAGUACAA-3'), miR-7 antisense oligonucleotide (miR-7 ASO, 5'-ACAACAAAAUCA CUAGUCUUCCA-3'), ASO NC (5'-CAGUACUUUUGUGUA GUACAA-3'), small interfering RNA (si)-NC (5'-UUCUCC GAACGUGUCACGUTT-3'), si-CDR1as (5'-CCAAUAAGG CCAGUUCAUUTT-3') were used at $50 \mathrm{mM}$. pcDNA3 (abbr. NC), pcDNA3-CDR1as (abbr. CDR1as), pcDNA3-CKAP4 were used at $25 \mathrm{ng} / \mathrm{ml}$ in six-well plates seeded with $3 \times 10^{5}$ VSMCs cells. Lipofectamine ${ }^{\circledR} 2000$ (Invitrogen; Thermo Fisher Scientific, Inc.) was used for all transfections according to the manufacturer's instructions. The transfected cells were used for subsequent experiment at $48 \mathrm{~h}$ post-transfection.

Reverse transcription-quantitative PCR (RT-qPCR) analysis. TRIzol $^{\circledR}$ (Invitrogen; Thermo Fisher Scientific, Inc.) was used for total RNA extraction from aortic specimens or VSMCs. Briefly, $1 \mu \mathrm{g}$ of the RNA was reverse transcribed using the High-Capacity cDNA reverse transcription kit (cat. no. 4368813; Thermo Fisher Scientific, Inc.). The temperature protocol for reverse transcription was as follows: Incubation at $25^{\circ} \mathrm{C}$ for $5 \mathrm{~min}, 37^{\circ} \mathrm{C}$ for $120 \mathrm{~min}$ and $85^{\circ} \mathrm{C}$ for $5 \mathrm{~min}$. qPCR was then performed using SYBR Green Master mix (Thermo Fisher Scientific, Inc.) using the following parameters: $95^{\circ} \mathrm{C}$ for $5 \mathrm{~min}$; 40 cycles of $10 \mathrm{sec}$ at $95^{\circ} \mathrm{C}, 20 \mathrm{sec}$ at $55^{\circ} \mathrm{C}$ and $15 \mathrm{sec}$ at $72^{\circ} \mathrm{C}$, followed by a melting curve of $10 \mathrm{sec}$ at $95^{\circ} \mathrm{C}, 60 \mathrm{sec}$ at $60^{\circ} \mathrm{C}$ and a continuous increase up to $95^{\circ} \mathrm{C}$ at a rate of $0.15^{\circ} \mathrm{C} / \mathrm{sec}$ for $15 \mathrm{sec}$ with a continuous reading of the fluorescence. The $2^{-\Delta \Delta \mathrm{Cq}}$ method was used to quantify the target gene expression (17). The primers used were as follows: miR-7 forward, 5'-CCACGT TGGAAGACTAGTGATTT-3' and reverse, 5'-TATGGTTGT TCTGCTCTCTGTCTC-3'; CDR1as forward, 5'-GTGTCT CCAGTGTATCGGCG-3' and reverse, 5'-TACTGGCACCAC TGGAAACC-3'; GAPDH forward, 5'-GACTCATGACCA CAGTCCATGC-3' and reverse, 5'-AGAGGCAGGGATGAT GTTCTG-3'; U6 forward, 5'-CTCGCTTCGGCAGCACA-3' and reverse, 5'-AACGCTTCACGAATTTGCGT-3'.

Bioinformatics analysis. StarBase v3.0 (http://starbase.sysu. edu.cn) was used for predicting the potential long ncRNA (lncRNA) that can bind miR-7. TargetScan 7.1 (http://www. targetscan.org/vert_72/) was used to predict the downstream target genes of miR-7.

Luciferase reporter assay. Briefly, $1.5 \times 10^{4}$ cells were seeded in a 24-well plate. After $24 \mathrm{~h}$, the cells were transfected with miR-7 mimics, miR-7 ASO, miR-NC or ASO NC as well as the pmirGLO vector (Promega Corporation), which was inserted with the wild-type (WT) 3'-untranslated region (UTR) of CKAP4 or a mutant (mut) form of CKAP4 3'-UTR which contained a mutated seed sequence (from 5'-GUCUUCC-3' into 5'-GUGUUGG-3'). At $48 \mathrm{~h}$ following transfection, the cells were used to measure the activities of firefly and Renilla luciferase using a Dual Luciferase reporter assay kit (Promega Corporation). The activity of Renilla luciferase was used as internal control.

Biotin pull-down assay. Cells were transfected with $50 \mu \mathrm{M}$ of biotinylated miR-7 mimic or miR-NC (as aforementioned; GeneCopoeia, Inc.) for $24 \mathrm{~h}$ and lysed in $500 \mu \mathrm{l}$ lysis buffer at $4^{\circ} \mathrm{C}$ for $30 \mathrm{~min}$, followed by the addition of $50 \mu \mathrm{l}$ blocked streptavidin magnetic beads and incubation for $4 \mathrm{~h}$ at $4^{\circ} \mathrm{C}$. TRIzol $^{\circledR}$ was used to extract the miR-7 that interacted with ncRNAs and the CDRlas expression levels were subsequently determined using RT-qPCR.

Western blot analysis. Following transfection for $48 \mathrm{~h}$, the VSMCs were lysed using RIPA buffer $(150 \mathrm{mM}$ Tris-HCl, 

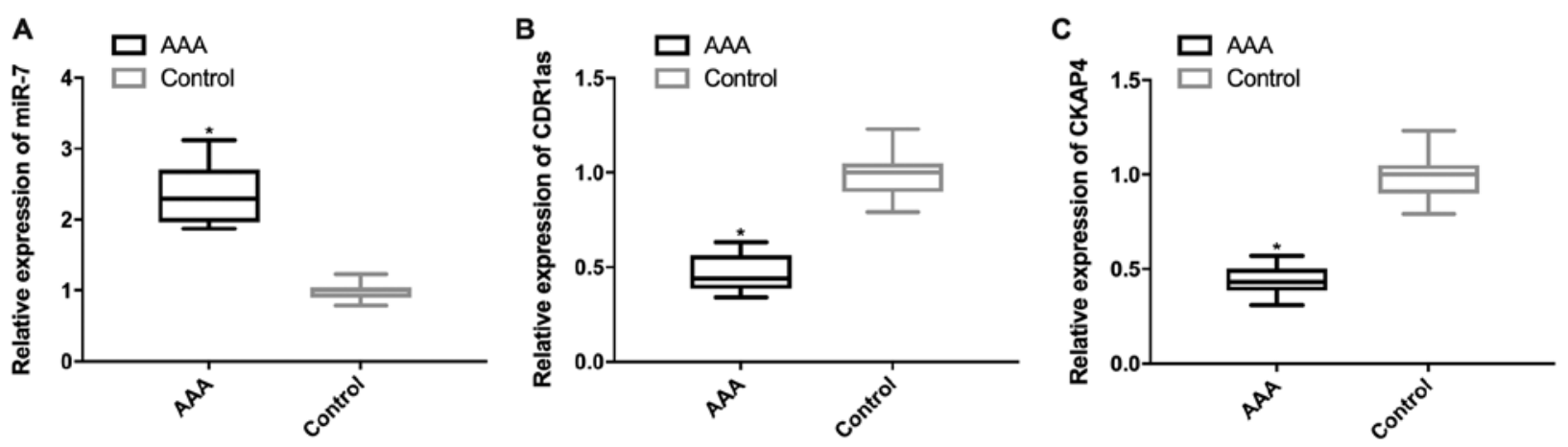

Figure 1. Verification of the expression levels of miR-7, CDR1as and CKAP4 mRNA in the aortic tissues of patients with AAA. Reverse transcription-quantitative PCR was performed to detect the expression levels of (A) miR-7, (B) CDR1as and (C) CKAP4. ${ }^{\mathrm{P}}<0.05$ vs. control). AAA, abdominal aortic aneurysm; CDR1as, cerebellar degeneration-related protein 1 antisense RNA; CKAP4, cytoskeleton-associated protein 4; miR, microRNA.

$50 \mathrm{mM} \mathrm{NaCl}, 1 \%$ Nonidet P-40 and 0.1\% Tween-20), followed by determining the protein concentration using Bradford assays (Bio-Rad Laboratories, Inc.). Proteins ( $30 \mu \mathrm{g}$ ) were separated by $10 \%$ SDS-PAGE, followed by transferring to PVDF membranes at $250 \mathrm{~mA}$ for $2.5 \mathrm{~h}$. After blocking for $1 \mathrm{~h}$ with $3 \% \mathrm{w} / \mathrm{v}$ skimmed milk at room temperature, the membranes were incubated with the following primary antibodies overnight at $4^{\circ} \mathrm{C}$ : Anti-CKAP4 (cat. no. ab84712; 1:2,000; Abcam), anti-cleaved-caspase 3 (cat. no. 9661; 1:1,000; Cell Signaling Technology, Inc.) and anti-GAPDH (cat. no. 2118; 1:10,000; Cell Signaling Technology, Inc.). Thenext day, the membranes were washed with Tris-buffered saline containing $0.1 \%$ Tween 20 , three times and incubated with a secondary antibody conjugated with horseradish peroxidase (cat. no. A0208, Beyotime Institute of Biotechnology) at 1:1,000 for $1 \mathrm{~h}$ at room temperature. Chemiluminescence was detected with an ECL kit (GE Healthcare Life Sciences), then quantified using ImageQuant software version 5.2 (GE Healthcare Life Sciences). GAPDH was used as an internal control.

Cell Counting Kit-8 (CCK-8) assay. To detect cell viability, a CCK-8 assay was performed. Treated and untreated VSMCs were cultured in a 96 -well plate at $3 \times 10^{4}$ cells/well. After $48 \mathrm{~h}$, 10\% CCK-8 solution (Dojindo Molecular Technologies, Inc.) was added to each well and incubated for $1 \mathrm{~h}$ at $37^{\circ} \mathrm{C}$, as per the manufacturer's instructions, followed by analysis using a microplate reader at a wavelength of $450 \mathrm{~nm}$.

Lactate dehydrogenase ( $L D H)$, reactive oxygen species (ROS) and caspase 3/7 detection assays. To detect the extent of VSMC apoptosis, LDH, ROS and caspase 3/7 assays were performed.

For the LDH assay, transfected cells were cultured at $5 \times 10^{3}$ cells/well in 96-well plates for $48 \mathrm{~h}$. After centrifuging at $350 \mathrm{x} \mathrm{g}$ for $5 \mathrm{~min}$ at $4^{\circ} \mathrm{C}$ and discarding of the supernatant, $150 \mu 1 \mathrm{LDH}$ release regent (cat. no. C0016; Beyotime Institute of Biotechnology) was added to each well and incubated for $1 \mathrm{~h}$ at $37^{\circ} \mathrm{C}$. The signal was detected using a microplate reader (Bio-Rad Laboratories, Inc.) at $490 \mathrm{~nm}$.

Intracellular ROS was detected using 2',7'-dichlorofluorescin diacetate (DCF-DA; Sigma-Aldrich; Merck KGaA) staining as previously described (18). After discarding the cell medium, the cells $\left(5 \times 10^{3}\right.$ cells/well in 96 -well plates) were stained with $20 \mu \mathrm{M}$ DCF-DA solution at $37^{\circ} \mathrm{C}$ in the dark for $30 \mathrm{~min}$, followed by the measurement of the signal with a fluorescence reader (Bio-Rad Laboratories, Inc.) at excitation/emission wavelengths of $488 / 525 \mathrm{~nm}$.

Caspase 3/7 activity was quantitated using a Caspase-Glo ${ }^{\circledR}$ 3/7 Assay kit (Promega Corporation) according to the manufacturer's instructions.

Statistical analysis. Data are presented as the mean \pm standard error of the mean. When comparing the AAA aortic tissues and normal controls, data are presented using box and whiskers plots. Statistical analysis was performed using GraphPad Prism 8 software (GraphPad Software, Inc.) with one-way ANOVAs followed by Tukey's test. $\mathrm{P}<0.05$ was considered to indicate a statistically significant difference. All experiments were repeated at least three times.

\section{Results}

miR-7 is upregulated, whereas CDRIas and CKAP4 are downregulated in AAA tissues. The results of RT-qPCR demonstrated that miR-7 was expressed at higher levels in AAA aortic tissues compared with the normal controls (Fig. 1A). The expression levels of CDR1as and CKAP4 were notably downregulated in AAA (Fig. 1B and C).

Effects of CDRlas and miR-7 on the function of primary VSMCs. Transfection efficiency of CDR1as overexpression plasmids and siRNA, and miR-7 mimics and ASO was evaluated using RT-qPCR. The results revealed that CDR1as overexpression increased CDR las RNA levels 2-fold, whereas si-CDR1as downregulated the expression of this RNA by 60\%. miR-7 mimics upregulated miR-7 expression 1.8-fold, whereas miR-7 ASO downregulated its expression by $70 \%$ (Fig. 2A). The results of the CCK-8 assay revealed that cell viability was increased following transfection with either CDR1as or miR-7 ASO, but decreased following transfection with siRNA CDR1as or miR-7 mimics, compared with their respective controls (Fig. 2B). Similarly, Cell growth curve assay showed that cell proliferative ability was increased following transfection with CDR1as or miR-7 ASO, but decreased following transfection with siRNA CDR1as or miR-7 mimics (Fig. 2C and D). In addition, CDR 1as and miR-7 ASO decreased the ROS generation and LDH activity, which was increased following transfection with siRNA CDR1as or 

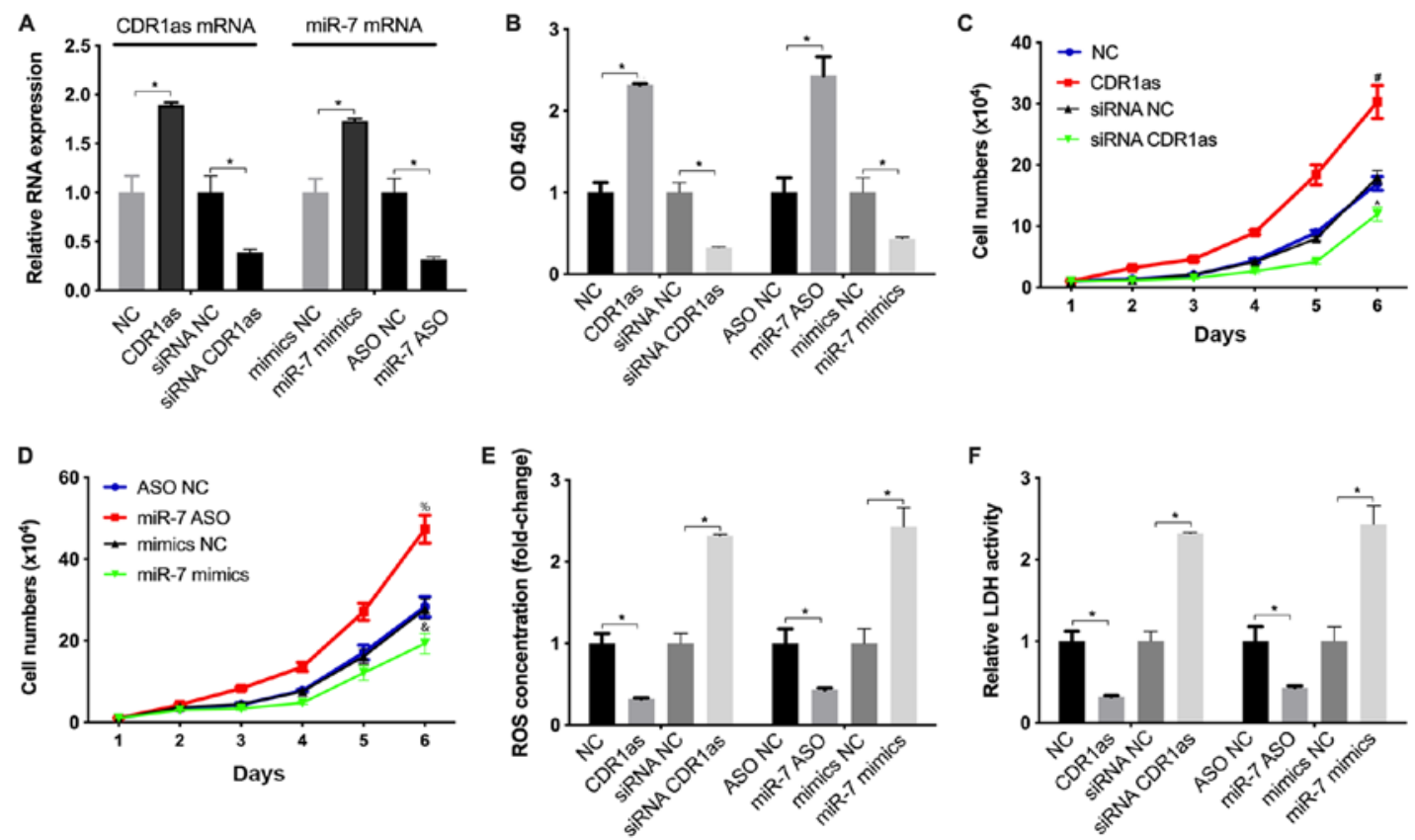

Figure 2. Effect of CDR1as and miR-7 on the phenotype of primary vascular smooth muscle cells. (A) The expression levels of CDR1as following transfection with a CDR1as overexpression vector and siRNA targeting CDR1as, as well as miR-7 following transfection with miR-7 ASO and mimics were detected by reverse transcription-quantitative PCR. (B) Cell viability was detected using Cell Counting Kit-8 assays. Cell growth curve assay was performed following transfections altering (C) CDRlas and (D) miR-7 expression levels. (E) The ROS generation and (F) the LDH activity were detected. ${ }^{*} \mathrm{P}<0.05 ;{ }^{*} \mathrm{P}<0.05, \mathrm{NC}$ vs. CDR1as; ${ }^{\wedge} \mathrm{P}<0.05$, siRNA NC vs. siRNA CDR1as; ${ }^{\%}$ ASO NC vs. miR-7 ASO, ${ }^{\circledR}$ mimics NC vs. miR-7 mimics). ASO, antisense nucleotide; CDR1as, cerebellar degeneration-related protein 1 antisense RNA; LDH lactate dehydrogenase; miR, microRNA; NC, negative control; ROS, reactive oxygen species; siRNA, small interfering RNA.

A
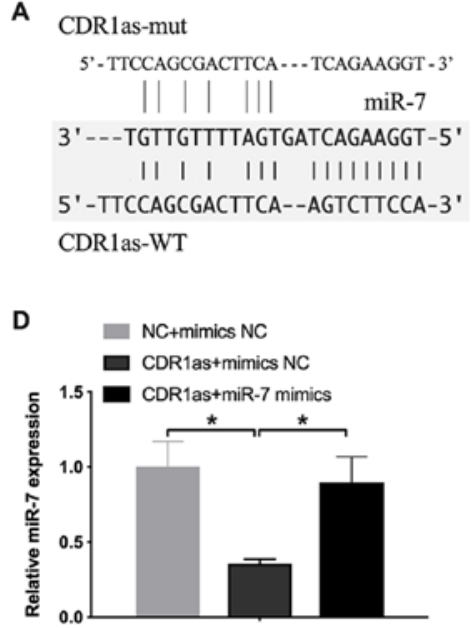

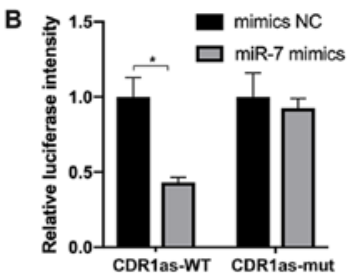

E

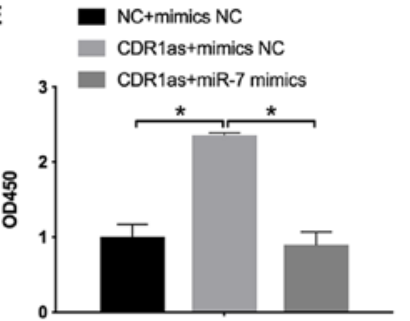

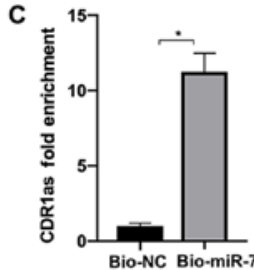

$\mathbf{F}$

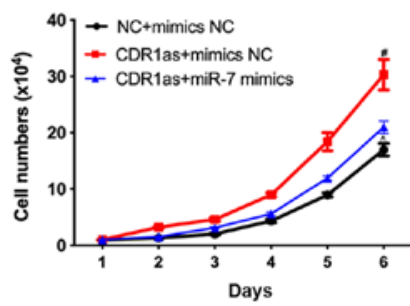

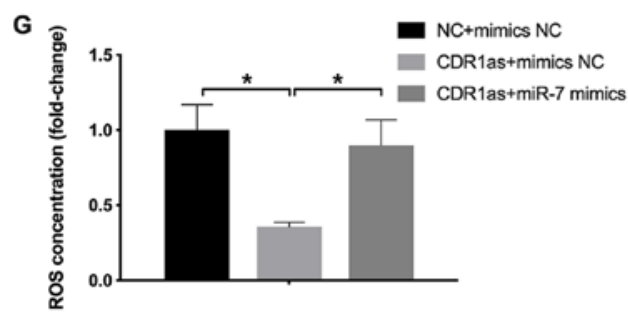

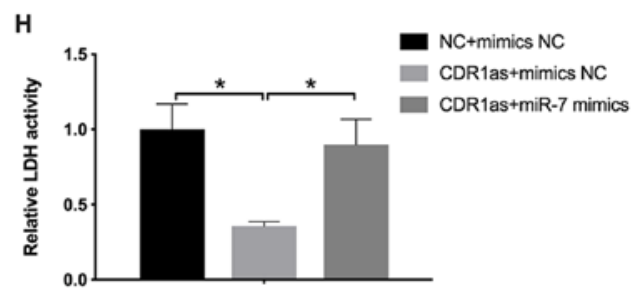

Figure 3. miR-7 mediates the effects of CDR1as. (A) Bioinformatics predicted the binding sites of CDR1as that are targeted by miR-7. (B) VSMCs were co-transfected with miR-7 mimics and CDR1as-WT or CDR1as-mut. (C) The pull-down assay was performed in cells transfected with Bio-miR-7 mimic or Bio-NC, followed by reverse transcription-quantitative PCR detection of CDR1as. (D) miR-7 expression levels were detected in VSMCs co-transfected with $\mathrm{NC}+$ mimics NC, CDR1as + mimics NC and CDR1as + miR-7 mimics. (E) Cell Counting Kit-8 assays were performed to detect cell viability. (F) Cell growth curves were produced to detect cell proliferation. (G) ROS concentration and (H) $\mathrm{LDH}$ activity were detected. ${ }^{*} \mathrm{P}<0.05$; ${ }^{\prime \prime} \mathrm{P}<0.05, \mathrm{NC}+$ mimics NC vs. CDR 1 as + mimics NC; ${ }^{\wedge} \mathrm{P}<0.05$, CDR1as + mimics NC vs. CDR1as + miR-7 mimics. Bio, biotinylated; CDR1as, cerebellar degeneration-related protein 1 antisense RNA; LDH, lactate dehydrogenase; miR, microRNA; mut, mutant sequence; NC, negative control; OD, optical density; ROS, reactive oxygen species; VSMCs, vascular smooth muscle cells; WT, wild-type sequence. 
miR-7 mimics, compared with the respective control groups (Fig. 2E and F). These results suggested that CDR1as and miR-7 exerted opposing effects on VSMCs.

Overexpression of miR-7 reverses the effects of CDRlas in VSMCs. The WT version of CDR1as was found to contain a potential binding site of miR-7 (Fig. 3A). To further investigate whether miR-7 can be sponged by CDR1as in VSMCs, a CDR1as luciferase reporter was constructed. The results demonstrated that miR-7 significantly reduced the luciferase intensity of CDR1as-WT, but had no significant effect on the luciferase intensity of CDR1as-mut (Fig. 3B). In addition, the biotin pull-down assay results revealed that CDR1as was pulled down in biotinylated miR-7-transfected cells by 11-fold compared with cells transfected with biotinylated miR-NC (Fig. 3C). As presented in Fig. 3D, miR-7 expression was suppressed by CDR1as in VSMCs, which could be reversed by co-transfection with miR-7 mimics. CCK-8 and cell proliferation curve assays revealed that the CDR1as-facilitated cell viability and proliferation was reversed by co-transfection with miR-7 (Fig. 3E and F). Additionally, CDRlas-mediated suppression of ROS generation and LDH activity was reversed by co-transfections with miR-7 (Fig. 3G and H). Therefore, these data suggested that miR-7 may be sponged by CDRlas and mediate the downstream effects of CDRlas in VSMCs.

CKAP4 serves as a direct target of miR-7. Bioinformatics analysis was performed using TargetScan to predict the potential target of miR-7, with CKAP4 being identified as a target for miR-7 (Fig. 4A). To confirm whether CKAP4 was regulated by miR-7, RT-qPCR and western blot analysis were performed, which demonstrated that overexpression of miR-7 notably downregulated the expression of CKAP4 compared with mimics NC, whereas knockdown of miR-7 upregulated the expression of CKAP4 compared with ASO NC (Fig. 4B and C). In addition, luciferase reporter assays confirmed that miR-7 mimics notably decreased the luciferase activity of CKAP4-3'UTR, whereas this was not observed with miR-7 ASO. The expression of miR-7 mimics and ASO had no significant effects on the luciferase activity of the CKAP4-3'UTR mut reporter plasmid (Fig. 4D). These data confirmed that CKAP4 was a direct target of miR-7 in VSMCs.

CDRlas/miR-7/CKAP4 axis is involved in the regulation of the apoptosis proteins, caspase-3/7. To investigate whether CDR1as/miR-7/CKAP4 regulated the function of VSMCs, the present study investigated the impact of miR-7 on CKAP4. Firstly, pcDNA3-CKAP4 was verified to have an effective transfection efficiency (Fig. 5A). It was then confirmed that miR-7-mediated suppression of CKAP4 could be rescued by transfection with pcDNA3-CKAP4 (Fig. 5B). miR-7 mediated induction of caspase $3 / 7$ activity can be rescued by co-transfection pcDNA3-CKAP4 with miR-7 mimics (Fig. 5C), which suggested that miR-7-induced apoptosis may be rescued by CKAP4. In addition, the results demonstrated that CDR1as upregulated the expression of CKAP4, which was reversed by co-transfection with CDRlas and miR-7 mimics (Fig. 5D). The CDR1as-reduced caspase 3/7 activity by was rescued following co-transfection with miR-7 mimics (Fig. 5E), suggesting that the CDR1as-mediated suppression

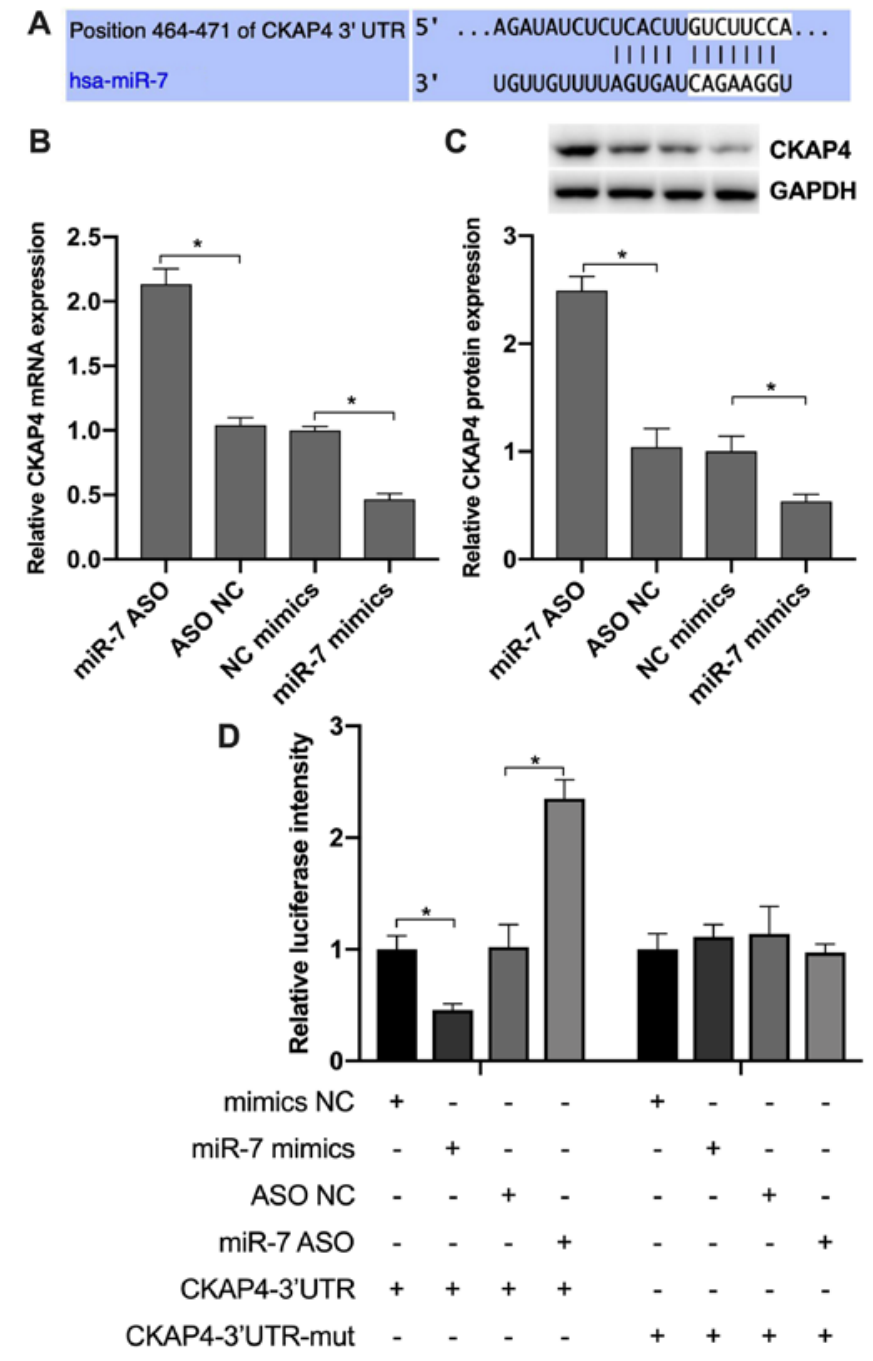

Figure 4. Validation of the direct interaction between miR-7 and CKAP4 (A) TargetScan analysis of the predicted interaction between miR-7 and CKAP4. (B) mRNA expression levels of CKAP4 were determined by reverse transcription-quantitative PCR. (C) The protein expression levels of CKAP4 were determined by western blotting. (D) The levels of luciferase activity following transfection of vascular smooth muscle cells with CKAP4 3'UTR-mut and 3'UTR-Wt sequences together with miR-7 mimics or ASO. ${ }^{*} \mathrm{P}<0.05$. ASO, antisense oligonucleotide; CKAP4, cytoskeleton-associated protein 4 ; miR, microRNA; mut, mutant; NC, negative control; UTR, untranslated region; Wt, wild-type.

of apoptosis was dependent on the downregulation of miR-7. In summary, CDR1as mediated the downregulation of miR-7, resulting in the upregulation of CKAP4 and leading to the inhibition of caspase 3/7 activity (Fig. 5F).

\section{Discussion}

A previous study has demonstrated that miR-7 can be sponged or inhibited by CDR1as in the developing midbrain of zebrafish, suggesting a newly identified mechanism of action for miRNA regulation (19). Further studies have confirmed that CDR1as promotes cell proliferation in hepatocellular carcinoma (15) and osteosarcoma (11) by suppressing miR-7 expression levels. The results of the present study demonstrated that CDR1as was expressed at a lower level, whereas miR-7 was upregulated in aortic tissue from patients with AAA compared with that of 
A

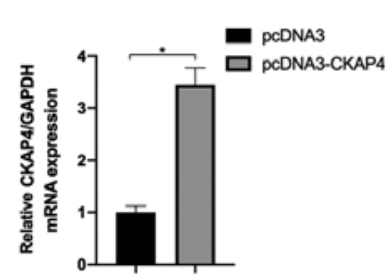

B

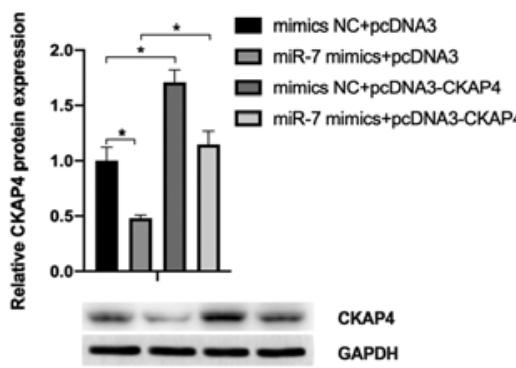

E

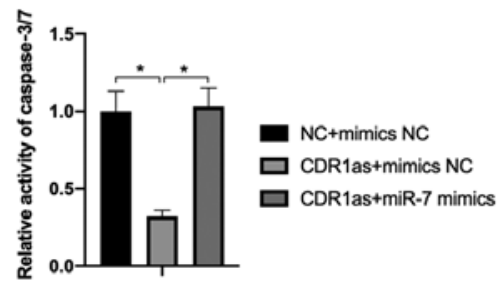

C

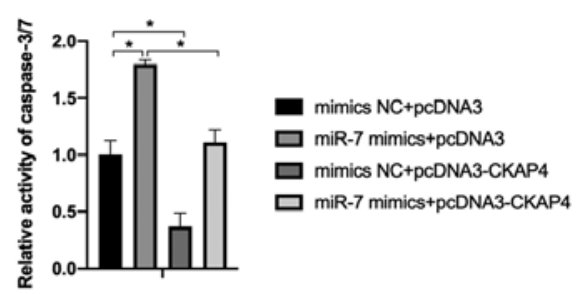

$\mathbf{F}$

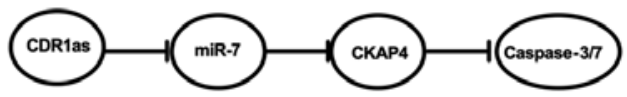

Figure 5. Detection of CKAP4 protein expression levels and caspase 3/7 activity. (A) Reverse transcription-quantitative PCR was used to detect the transfection efficiency of pcDNA3-CKAP4. VSMCs were co-transfected with miR-NC + pcDNA3, miR-7 mimics + pcDNA3, miR-NC + pcDNA3-CKAP4 and miR-7 mimics + pcDNA3-CKAP4, and the (B) relative protein expression levels of CKAP4 as well as the (C) activity of caspase 3/7 were measured. VSMCs were co-transfected with NC + miR-NC, CDR1as + miR-NC and CDR1as + miR-7 mimics, and the (D) relative protein expression levels of CKAP4 as well as the (E) activity of caspase 3/7 were measured. (F) The major pathway identified in this present study was that CDR1as-mediated suppression of miR-7, resulting in the upregulation of CKAP4. This ultimately led to the inhibition of caspase 3/7 activity. ${ }^{*} \mathrm{P}<0.05$. CDR1as, cerebellar degeneration-related protein 1 antisense RNA; CKAP4, cytoskeleton-associated protein 4; miR, microRNA; NC, negative control; VSMCs, vascular smooth muscle cells; NC, negative control.

normal tissue. In addition, the predicted miR-7 target CKAP4 was downregulated in AAA tissue compared with normal tissue. A previous study has identified that AAAs are associated with apoptosis of VSMCs (20). Therefore, the upregulated miR-7 and the downregulated CDR1as and CKAP4 may affect apoptosis in VSMCs.

Primary VSMCs were used in the present study to investigate the mechanism of action of CDRlas/miR-7, aiming to identify the mechanisms behind the pathogenesis in AAAs. In a myocardial infarction (MI) study, CDRlas promoted MI injuries by reducing miR-7a activity (21). CDR1as is also downregulated and miR-7 is upregulated in patients with Alzheimer's disease (22). In tumorigenesis, CDR las commonly serves as an oncogene in hepatocellular carcinoma (15), osteosarcoma (11) and non-small cell lung cancer (23). The results of the present study suggested that CDR1as may promote the proliferation and suppress the ROS generation and LDH activity of VSMCs, whereas co-expression of miR-7 can reverse the CDR1as-mediated effects. In addition, the results suggested that CDR1as may directly bind miR-7 in VSMCs. A previous study has demonstrated that ROS dysregulates the function of the extracellular matrix, thus promoting the apoptosis of VSMCs (24), which suggested that the CDR1as/miR-7 axis may contribute to the remodeling of AAA.

Studies on miR-7 have been conducted in different types of tumors, and its function as a tumor suppressor has been demonstrated in pancreatic cancer (25) and glioma (26). In VSMCs derived from AAA in the present study, miR-7 suppressed proliferation and promoted the generation of ROS as well as LDH activity. In addition, CKAP4 was confirmed to be a direct target for miR-7. Overexpression of CKAP4 reversed the miR-7-mediated effects on VSMCs. As the receptor of dickkopf-related protein 1 (DKK1), CKAP4 mediates downstream signaling to promote cellular proliferation and inhibit apoptosis through the activation of the PI3K/AKT pathway (27). Apoptosis of VSMCs is crucial for normal vascular remodeling (28). The PI3K/AKT signaling pathway is implicated in the proliferation of VSMCs, thus modulating vascular remodeling by decreasing the pro-apoptotic functions of Bcl2-associated death promoter $(29,30)$.

The results of the present study demonstrated that CKAP4 suppressed the activity of caspase $3 / 7$ and that miR-7-mediated increase in caspase $3 / 7$ activity could be reversed by the overexpression of CKAP4 in VSMCs. In addition, CDRlas-suppressed caspase $3 / 7$ activity was rescued by miR-7 overexpression. Thus, the activation of CDR1as in AAA may result in the downregulation of miR-7 and upregulation of CKAP4. This in turn may activate the PI3K/AKT pathway and result in the interference in the function of DKK1, leading to VMSC remodeling.

In conclusion, the results of the present study identified the functions of the CDR1as/miR-7/CKAP4 axis in VSMCs from patients with AAA and demonstrated that upregulated CDR1as served as an inhibitor of miR-7. This increased the expression of CKAP4 to facilitate the proliferation and suppress the apoptosis of VSMCs, leading to VSMC remodeling and progression of AAA. This newly identified mechanism may provide novel options for the treatment of AAA.

\section{Acknowledgements}

Not applicable. 


\section{Funding}

The current study was supported by Tianjin Commission of Science and Technology, grant no. 18ZXDBSY00160 (grant title: The treatment of aortic dissection in aged population of Tianjin) and grant. no. 16ZXMJSY00160 (grant title: The clinical research on Transcatheter Aortic Valve Implantation).

\section{Availability of data and materials}

The datasets used and/or analyzed during the current study are available from the corresponding author on reasonable request.

\section{Authors' contributions}

FZ and TC performed the experiments, FZ wrote the manuscript. NJ designed the experiments. All authors read and approved the final manuscript.

\section{Ethics and approval}

Ethics and approval were obtained from the Institutional Review Board of Tianjin Chest Hospital. All patients signed written informed consent.

\section{Patient consent for publication}

Not applicable.

\section{Competing interests}

The authors declare that they have no competing interests.

\section{References}

1. Scott RA, Bridgewater SG and Ashton HA: Randomized clinical trial of screening for abdominal aortic aneurysm in women. Br J Surg 89: 283-285, 2002.

2. Reimerink JJ, van der Laan MJ, Koelemay MJ, Balm R and Legemate DA: Systematic review and meta-analysis of population-based mortality from ruptured abdominal aortic aneurysm. Br J Surg 100: 1405-1413, 2013.

3. Cao X, Cai Z, Liu J, Zhao Y, Wang X, Li X and Xia H: miRNA-504 inhibits p53-dependent vascular smooth muscle cell apoptosis and may prevent aneurysm formation. Mol Med Rep 16: 2570-2578, 2017.

4. Chaikof EL, Brewster DC, Dalman RL, Makaroun MS, Illig KA, Sicard GA, Timaran CH, Upchurch GR Jr and Veith FJ; Society for Vascular Surgery: The care of patients with an abdominal aortic aneurysm: The society for vascular surgery practice guidelines. J Vasc Surg 50 (4 Suppl): S2-S49, 2009.

5. Ding L, Ren J, Zhang D, Li Y, Huang X, Ji J, Hu Q, Wang H, Ni Y and Hou Y: The TLR3 agonist inhibit drug efflux and sequentially consolidates low-dose cisplatin-based chemoimmunotherapy while reducing side effects. Mol Cancer Ther 16 : 1068-1079, 2017.

6. Bartel DP: MicroRNAs: Genomics, biogenesis, mechanism, and function. Cell 116: 281-297, 2004.

7. Yang Z, Xie L, Han L, Qu X, Yang Y, Zhang Y, He Z, Wang Y and Li J: Circular RNAs: Regulators of cancer-related signaling pathways and potential diagnostic biomarkers for human cancers. Theranostics 7: 3106-3117, 2017.

8. Zhao ZJ and Shen J: Circular RNA participates in the carcinogenesis and the malignant behavior of cancer. RNA Biol 14: 514-521, 2017.

9. Lasda E and Parker R: Circular RNAs: Diversity of form and function. RNA 20: 1829-1842, 2014.
10. Memczak S, Jens M, Elefsinioti A, Torti F, Krueger J, Rybak A, Maier L, Mackowiak SD, Gregersen LH, Munschauer M, et al: Circular RNAs are a large class of animal RNAs with regulatory potency. Nature 495: 333-338, 2013.

11. Xu B, Yang T, Wang Z, Zhang Y, Liu S and Shen M: CircRNA CDR1as/miR-7 signals promote tumor growth of osteosarcoma with a potential therapeutic and diagnostic value. Cancer Manag Re 10: 4871-4880, 2018.

12. Chan CYT, Cheuk BLY and Cheng SWK: Abdominal aortic aneurysm-associated MicroRNA-516a-5p regulates expressions of methylenetetrahydrofolate reductase, matrix metalloproteinase-2, and tissue inhibitor of matrix metalloproteinase-1 in human abdominal aortic vascular smooth muscle cells. Ann Vasc Surg 42: 263-273, 2017.

13. Kimura H, Fumoto K, Shojima K, Nojima S, Osugi Y, Tomihara H, Eguchi H, Shintani Y, Endo H, Inoue M, et al: CKAP4 is a Dickkopf1 receptor and is involved in tumor progression. J Clin Invest 126: 2689-2705, 2016.

14. Sun CM, Geng J, Yan Y, Yao X and Liu M: Overexpression of CKAP4 is associated with poor prognosis in clear cell renal cell carcinoma and functions via cyclin B signaling. J Cancer 8: 4018-4026, 2017.

15. Yajima N, Masuda M, Miyazaki M, Nakajima N, Chien S and Shyy JY: Oxidative stress is involved in the development of experimental abdominal aortic aneurysm: A study of the transcription profile with complementary DNA microarray. J Vasc Surg 36: 379-385, 2002.

16. Liang B, Wang S, Wang Q, Zhang W, Viollet B, Zhu Y and Zou MH: Aberrant endoplasmic reticulum stress in vascular smooth muscle increases vascular contractility and blood pressure in mice deficient of AMP-activated protein kinase- $\alpha 2$ in vivo. Arterioscler Thromb Vasc Biol 33: 595-604, 2013.

17. Livak KJ and Schmittgen TD: Analysis of relative gene expression data using real-time quantitative PCR and the 2(-Delta Delta C(T)) method. Methods 25: 402-408, 2001.

18. Yu L, Gong X, Sun L, Zhou Q, Lu B and Zhu L: The circular RNA Cdrlas act as an oncogene in hepatocellular carcinoma through targeting miR-7 expression. PLoS One 11: e0158347, 2016.

19. Stather PW, Sylvius N, Sidloff DA, Dattani N, Verissimo A, Wild JB, Butt HZ, Choke E, Sayers RD and Bown MJ: Identification of microRNAs associated with abdominal aortic aneurysms and peripheral arterial disease. Br J Surg 102: 755-766, 2015.

20. Su Z, Yang Z, Xu Y, Chen Y and Yu Q: MicroRNAs in apoptosis, autophagy and necroptosis. Oncotarget 6: 8474-8490, 2015.

21. Geng HH, Li R, Su YM, Xiao J, Pan M, Cai XX and Ji XP: The circular RNA Cdrlas promotes myocardial infarction by mediating the regulation of miR-7a on its target genes expression. PLoS One 11: e0151753, 2016.

22. Kumar L, Shamsuzzama, Haque R, Baghel T and Nazir A: Circular RNAs: The emerging class of non-coding RNAs and their potential role in human neurodegenerative diseases. Mol Neurobiol 54: 7224-7234, 2017.

23. Zhang X, Yang D and Wei Y: Overexpressed CDR1as functions as an oncogene to promote the tumor progression via miR-7 in non-small-cell lung cancer. Onco Targets Ther 11: 3979-3987, 2018.

24. Emeto TI, Moxon JV, Au M and Golledge J: Oxidative stress and abdominal aortic aneurysm: Potential treatment targets. Clin Sci (Lond) 130: 301-315, 2016.

25. Xia J, Cao T, Ma C, Shi Y, Sun Y, Wang ZP and Ma J: miR-7 suppresses tumor progression by directly targeting MAP3K9 in pancreatic cancer. Mol Ther Nucleic Acids 13: 121-132, 2018.

26. Visani M, de Biase D, Marucci G, Cerasoli S, Nigrisoli E, Bacchi Reggiani ML, Albani F, Baruzzi A and Pession A; PERNO study group: Expression of 19 microRNAs in glioblastoma and comparison with other brain neoplasia of grades I-III. Mol Oncol 8: 417-430, 2014

27. Tuffy KM and Planey SL: Cytoskeleton-associated protein 4: Functions beyond the endoplasmic reticulum in physiology and disease. ISRN Cell Biol 2012: 1-11, 2012.

28. McCabe CD, Spyropoulos DD, Martin D and Moreno CS: Genome-wide analysis of the homeobox C6 transcriptional network in prostate cancer. Cancer Res 69: 1988-1996, 2008.

29. Jang SW, Liu X, Fu H, Rees H, Yepes M, Levey A and Ye K: Interaction of Akt-phosphorylated SRPK2 with 14-3-3 mediates cell cycle and cell death in neurons. J Biol Chem 284: 24512-24525, 2009.

30. Wang S, Wang Y, Jiang J, Wang R, Li L, Qiu Z, Wu H and Zhu D: 15-HETE protects rat pulmonary arterial smooth muscle cells from apoptosis via the PI3K/Akt pathway. Prostaglandins Other Lipid Mediat 91: 51-60, 2010.

This work is licensed under a Creative Commons Attribution-NonCommercial-NoDerivatives 4.0 International (CC BY-NC-ND 4.0) License. 